Annuaire suisse de politique de développement

$17 \mid 1998$

Propriété intellectuelle : quels enjeux pour les pays en développement ?

\title{
Accès aux médicaments et accord TRIPS
}

\section{Pascale Brudon}

\section{(2) OpenEdition}

\section{Journals}

Édition électronique

URL : http://journals.openedition.org/aspd/762

DOI : 10.4000/aspd.762

ISSN : 1663-9669

Éditeur

Institut de hautes études internationales et du développement

\section{Édition imprimée}

Date de publication : 1 avril 1998

Pagination : 85-94

ISSN : 1660-5934

\section{Référence électronique}

Pascale Brudon, "Accès aux médicaments et accord TRIPS », Annuaire suisse de politique de développement [En ligne], 17 | 1998, mis en ligne le 31 juillet 2012, consulté le 08 septembre 2020. URL : http://journals.openedition.org/aspd/762 ; DOI : https://doi.org/10.4000/aspd.762 


\title{
ACCÈS AUX MÉDICAMENTS ET ACCORD TRIPS
}

\author{
PASCALE BRUDON*
}

$\mathrm{M}$

ALGRÉ une amélioration régulière et constante de la santé dans le monde, y compris dans la plupart des pays en développement, chaque année 17 millions de personnes dont une majorité d'enfants de moins de 5 ans meurent de maladies transmissibles qui pourraient souvent être évitées par une politique active de prévention, ou traitées et guéries par une thérapeutique appropriée (maladies infectieuses et parasitaires). Du fait d'un vieillissement rapide de la population dans les pays développés, mais également dans les pays en développement, un nombre croissant de personnes sont atteintes de maladies non transmissibles chroniques qui nécessitent des traitements prolongés. De nouvelles maladies - les maladies émergentes - ont fait leur apparition ces dernières années, tel le sida; d'autres, connues depuis longtemps et parfois même contrôlées, voire éradiquées, regagnent du terrain dans de nombreux pays (la tuberculose, le paludisme, etc.). Enfin, la résistance croissante de nombreux agents infectieux (bactéries, parasites) responsables des pathologies dominantes (paludisme, infections respiratoires aiguës, tuberculose, etc.) dans les pays en développement et des infections nosocomiales dans les pays développés pose aujourd'hui des problèmes graves.

Dans un tel contexte épidémiologique, si la prévention est plus que jamais l'outil majeur pour lutter efficacement contre la maladie, les médicaments continuent de jouer un rôle essentiel pour contrôler, voire réduire l'extension des pathologies dominantes et améliorer ainsi l'espérance de vie sans incapacité, et la qualité de la vie. Or dans le domaine du médicament, comme dans beaucoup d'autres, les inégalités sont considérables entre les pays développés et le reste du monde. La consommation de médicaments, qui s'est élevée en 1995 à 284 milliards de dollars (prix producteur), est concentrée dans les pays développés puisque cette année-là, $80 \%$ des médicaments produits dans le monde ont été consommés par $25 \%$ de la population mondiale, celle qui vit en Amérique du Nord, en Europe et au Japon'. Dans les pays en développement au contraire, plus d'un milliard de personnes (probablement deux) n'ont pas un accès régulier aux médicaments les plus essentiels ${ }^{2}$. Depuis dix ans, les nouveaux médicaments - et ils sont nombreux - ont été pour la plupart découverts, produits et commercialisés par un petit nombre de sociétés pharmaceutiques transnationales situées pour l'essentiel dans les pays industrialisés ${ }^{3}$.

En fait, bien qu'une telle présentation soit un peu schématique et donc forcément incomplète, il apparaît commode pour la compréhension de la situation pharmaceutique dans le monde de séparer les pays en trois grands groupes: les

* Fonctionnaire scientifique, Programme d'action pour les médicaments essentiels, Organisation mondiale de la santé, Genève.

1. Scrip. $\mathrm{n}^{\circ} 2172$, October $15,1996, \mathrm{p} .14$.

2. Rapport sur la santé dans le monde 1996, OMS, Genève. p. 84.

3. Ballance R., Pogány J., Forstner H., The World's Pharmaceutical Industries: An International Perspective on Innovation, Competition and Policy, Vienna, United Nations Industrial Development Organization (UNIDO), 1992. 
pays industrialisés, où sont concentrées la recherche et la production et qui consomment à eux seuls plus des quatre cinquièmes (en valeur) des médicaments produits dans le monde, quelques pays en développement dont la population est en général importante (Chine, Inde, Mexique, Brésil, Argentine) et qui ont réussi à développer une industrie pharmaceutique nationale, voire même une certaine capacité de recherche, et enfin les pays purement consommateurs, qui fabriquent quelques produits finis mais importent la majorité de leurs médicaments.

Dans le cadre de l'Uruguay Round et en annexe à la convention-cadre de l'OMC figure un accord d'une importance particulière pour les médicaments: l'Accord général sur les aspects des droits de propriété intellectuelle liés au commerce (accord TRIPS), dont les objectifs consistent à renforcer et à harmoniser à l'échelle mondiale la protection de la propriété intellectuelle, comprenant la propriété industrielle.

Qu'en sera-t-il demain de la situation pharmaceutique des pays en développement après la mise en œuvre de l'accord TRIPS ? Les politiques pharmaceutiques nationales et les stratégies qui ont pour objectif principal d'améliorer la disponibilité des médicaments et qui sont soutenues par les organismes internationaux pourront-elles continuer à se développer? L'accord conduira-t-il à des stratégies qui permettront une meilleure accessibilité aux médicaments essentiels, anciens ou nouveaux, dans les pays en développement et favoriseront le transfert de technologie? Ou, au contraire, cet accord constituera-t-il un fardeau supplémentaire pour les budgets de santé déjà déficitaires des pays en développement et pour les populations de ces pays? Finalement, l'accord TRIPS est-il favorable à une meilleure couverture pharmaceutique dans les pays en développement? Bien que les choses n'en soient qu'à leur début, il paraît nécessaire d'examiner les effets possibles de cet accord sur les politiques pharmaceutiques des pays en voie de développement (PVD) afin de proposer une évolution des stratégies destinées à augmenter la disponibilité de médicaments essentiels de qualité à un coût abordable.

\section{POLITIQUES PHARMACEUTIQUES ET PROTECTION INTELLECTUELLE}

\subsection{PAYS INDUSTRIALISÉS}

Le système des brevets pour les produits pharmaceutiques a évolué au cours des temps. Plus les pays industrialisés se développaient, plus leur système était strict. La plupart des pays développés ont des régimes de brevets assez stricts, en général instaurés dans les années 70. Le système de protection des Etats-Unis est celui qui protège au mieux les intérêts de l'inventeur: vingt ans de protection de brevet aussi bien pour les procédés que pour les produits, pas d'obligations pour les titulaires de brevets. Ces derniers peuvent utiliser ou non leur brevet, le donner sous licence ou pas. Le consensus n'est cependant pas absolu au sein de ces pays; certains groupes de patients, par exemple les personnes âgées aux Etats-Unis, sont opposés à des protections trop longues qui empêchent la commercialisation de produits moins chers. Mais la tendance dans les pays industrialisés est à un renforcement et une extension de la période de protection des 
brevets. Les raisons de cette évolution résident probablement dans le fait que les brevets sont des instruments fondamentaux qui permettent aux firmes pharmaceutiques faisant de la recherche de récupérer leurs investissements ${ }^{4}$. Les brevets permettent des prix élevés fournissant à leur tour des profits pour continuer à faire de la recherche qui à son tour produit de nouveaux médicaments. Or, dans les pays développés la durée effective de protection des médicaments a diminué du fait des réglementations imposées pour commercialiser un produit. De plus la compétition a augmenté avec l'arrivée sur le marché de producteurs de médicaments génériques. Enfin, le marché des pays en développement, tout au moins de certains d'entre eux, commence à devenir intéressant. De ce fait la restauration d'une protection effective par les brevets dans les pays développés et l'instauration d'une protection plus efficace dans les pays en développement font partie des stratégies légitimes des firmes pharmaceutiques transnationales pour ne pas perdre de parts de marché et pour continuer à mener des activités de recherche de plus en plus coûteuses ${ }^{5}$.

\subsection{PAYS EN DÉVELOPPEMENT AYANT UNE INDUSTRIE PHARMACEUTIQUE NATIONALE}

Dès la fin de la Seconde Guerre mondiale, de grands pays tels que l'Argentine, le Brésil, l'Egypte, l'Inde, le Mexique et la République de Corée mirent en place des politiques d'incitation à la production locale de médicaments en vue d'augmenter leur consommation sur le marché intérieur. La plupart d'entre eux ont pu accroître leur offre de médicaments nouveaux à des prix abordables par le biais d'une protection intellectuelle faible. Aucun de ces pays n'a cependant pu découvrir de nouveaux médicaments. L'Argentine et le Brésil n'avaient pas de brevets pour les produits pharmaceutiques. Les firmes pouvaient copier et vendre sur le marché national les produits pharmaceutiques inventés par d'autres firmes sans violer la loi nationale. Une position intermédiaire adoptée par nombre de pays en développement était d'avoir seulement des brevets de produits ou seulement des brevets de procédés. Un inventeur pouvait breveter le procédé par lequel il fabriquait un produit mais pas le médicament lui-même; une autre firme avait alors la possibilité de synthétiser le même médicament si elle était capable de développer un autre mode de fabrication. Les pays jouaient aussi sur la période de durée des brevets qui pouvait être plus ou moins longue et sur la possibilité de mettre en place des licences obligatoires (le titulaire du brevet doit fournir des licences non exclusives à ceux qui souhaitent utiliser le brevet en échange de redevances raisonnables). Enfin, par le biais de l'obligation d'exploiter le brevet, un titulaire de brevet pouvait être obligé de produire le nouveau médicament dans le pays qui imposait cette condition sinon il perdait son brevet dans ce pays.

Ainsi le Indian Patents Act édicté en 1970 supprima les brevets pour les produits dans les domaines liés à la santé et limita la durée de protection des procédés entre cinq et sept ans pour les médicaments. La loi permit aussi l'utilisation de licences obligatoires pour les articles qui avaient un intérêt pour la santé publique. Cette politique porta ses fruits. Des firmes domestiques produisirent

4. Nogues J., «Social costs and benefits of introducing patent protection for pharmaceutical drugs in developing countries», The Developing Economies, March 1993, vol. 31 (1), pp. 24-53.

5. Nogues J., «Patents and pharmaceutical drugs: Understanding the pressures on developing countries », Journal of World Trade, 1990, vol. 24 (6), pp. 81-104. 
dès 1979 des médicaments qui avaient reçu des brevets à l'extérieur. En 1990 au moins une douzaine de firmes indiennes étaient capables de produire des médicaments relativement nouveaux tels que la ciprofloxacine (antibiotique) ou la ranitidine (médicament antiulcéreux) à partir de procédés de fabrication différents des procédés utilisés par l'inventeur du produit. Les prix de ces médicaments étaient beaucoup plus bas que les prix dans le reste du monde. Par exemple pour la ranitidine le prix en Inde était 16,6 fois plus bas que le prix auquel le médicament était vendu par Glaxo (inventeur du produit) en GrandeBretagne $^{6}$.

De même, en République de Corée, la Shin Poong Pharmaceutical Company synthétisa, en 1983, le praziquantel, médicament découvert par deux compagnies pharmaceutiques allemandes: Bayer et E. Merck, breveté en Allemagne en 1973 et largement commercialisé sur le marché international au début des années 80 . Le praziquantel représente la découverte la plus importante dans le traitement de la schistosomiase, maladie endémique dans plus de 70 pays, qui affecte plus de 200 millions de personnes dans le monde. Il est aussi le traitement de choix pour d'autres maladies dont la douve de Chine (opisthorchiase). Au début des années $80,10 \%$ de la population coréenne souffraient de la douve de Chine. Le médicament, mis sur le marché par Bayer, était financièrement peu accessible aux malades qui provenaient essentiellement des couches les plus défavorisées de la population. La Shin Poong Pharmaceutical Company reçut donc un financement du gouvernement coréen pour développer avec un institut de recherche public un nouveau procédé de fabrication, ce qui fut fait pour une somme relativement modeste (14'000 dollars). Ce nouveau procédé avait aussi l'avantage d'entraîner des coûts de fabrication inférieurs. Le produit fut breveté en Corée en 1985 et obtint un brevet de procédé dans 12 pays dont les EtatsUnis, le Japon et la Grande-Bretagne. Ce développement permit:

- Une réduction des prix du praziquantel sur le marché coréen grâce à une augmentation de la concurrence et de la demande. En 1983, le prix d'un comprimé commercialisé par la Shin Poong Pharmaceutical Company sous le nom de Distocide ${ }^{\circledast}$ était de 3,22 dollars contre 4,83 pour Bayer; en 1994, en prix réel le prix d'un comprimé de la Shin Poong Pharmaceutical Company était de 2,03 dollars et celui de Bayer de 1,84 dollar.

a Une augmentation des exportations de praziquantel sous forme de matière première ou de produit fini, notamment en Chine, pour le traitement de masse de la population des zones endémiques mais aussi dans des pays développés (Suisse, Pays-Bas, etc.).

- La production de praziquantel au Soudan et en Egypte par le biais d'accords entre des firmes nationales et la Shin Poong Pharmaceutical Company.

๖ Des bénéfices non négligeables en termes de santé pour de nombreux habitants de la planète ${ }^{7}$.

6. Bidwai P., «One step forward, many steps backward: dismemberment of India's national drug policy», Development Dialogue, 1995, vol. 1, pp. 193-222.

7. Reich M.R. et al., International strategies for tropical disease treatments: Experiences with praziquantel. WHO/DAP/CTD/98.5, Genève, OMS, 1998. 


\subsection{AUTRES PAYS EN DÉVELOPPEMENT}

Dans ces pays, la principale politique qui a été mise en œuvre à partir du début des années 80 sous l'égide de l'OMS est celle des «médicaments essentiels»: en 1977, des experts réunis par l'OMS ont développé une liste modèle de médicaments qui ont été appelés médicaments essentiels et qui permettent de traiter la majorité des maladies ${ }^{8}$. Cette liste, remise à jour régulièrement, contient entre 250 et 300 médicaments sous dénomination commune internationale; les pays peuvent la modifier en fonction de leur propre pathologie, de leurs services de santé, de la formation de leur personnel, etc. Cette sélection des médicaments, accompagnée de mesures telles que des approvisionnements faits par appels d'offres et sous nom générique, des interventions pour stimuler la concurrence par les prix et la vente de médicaments génériques, une meilleure formation des prescripteurs et du public à l'usage des médicaments, etc., permet de rationaliser le secteur pharmaceutique et de soigner les populations au meilleur coût. Plus de 100 pays ont maintenant une liste de médicaments essentiels, dont la plupart sont des médicaments qui ont subi l'épreuve du temps et qui ne sont donc en général plus protégés par des brevets; ils peuvent être achetés sur le marché international à des prix très compétitifs. Néanmoins la majorité des listes de médicaments essentiels des pays en développement contiennent aussi des médicaments relativement nouveaux et encore protégés par des brevets. Le nombre de ces nouveaux médicaments essentiels ne peut qu'augmenter dans les années à venir du fait de maladies telles que le sida (médicaments antirétroviraux) et de la résistance des micro-organismes aux anciens médicaments, notamment aux antibiotiques. De nombreux pays en développement ont profité de la faiblesse de la protection intellectuelle ou de l'absence d'un mécanisme global de protection pour importer ces nouveaux médicaments de pays qui les fabriquent avec des procédés de fabrication différents de celui de l'inventeur et qui les vendent moins cher.

Ainsi, les pays en développement, quel que soit leur niveau socio-économique, ont tenté d'améliorer l'accès aux médicaments pour leur population en utilisant entre autres la faible protection accordée aux inventions dans le monde. On comprend mieux pourquoi l'accord TRIPS a été l'occasion d'une longue bataille entre les pays développés et les pays en développement, les premiers voulant protéger leur industrie et sa capacité de recherche, les seconds voulant développer une capacité nationale et améliorer la disponibilité des médicaments. En 1994, la plupart des pays ont amendé leur législation et augmenté la protection de la propriété intellectuelle pour les médicaments. Cela a eu lieu en grande partie sous la pression des pays développés; l'Argentine, le Brésil, l'Inde ou la Thaïlande ont fait l'objet d'enquêtes ou de mesures de rétorsion dans le cadre de la section 301 du «Trade and Tariffs Act» de la législation américaine?

8. La sélection des médicaments essentiels. Rapport d'un comité d'experts de l'OMS, Série de rapports techniques $\mathrm{n}^{\circ}$ 615. Genève, OMS, 1977.

9. Correa C.M., The Uruguay Round and drugs, WHO Task Force on Health Economics, Geneva, WHO, 1997. 


\section{ACCORD TRIPS ET PRODUITS PHARMACEUTIQUES: QU'EST-CE QUI VA CHANGER?}

L'accord TRIPS contient des clauses importantes pour la situation pharmaceutique dans les pays en développement ${ }^{10}$, notamment:

- Tout Etat membre de l'OMC devra accorder une protection par brevet, de produit ou de procédé, à toute invention dans le domaine pharmaceutique (article 27.1), ce qui signifie que toute imitation de médicament breveté est dorénavant interdite sous peine de sanctions commerciales imposées par l'organe de règlement des différends de l'OMC. Le même article stipule que le traitement des droits de brevets doit être identique quels que soient le lieu d'origine de l'invention, le domaine technologique ou le fait que les produits sont importés ou produits sur place. Cette dernière condition ne permettra plus aux pays de délivrer des licences obligatoires lorsqu'une firme n'exploitait pas le brevet. De même un pays ne pourra plus obliger une entreprise étrangère qui détenait un brevet à produire le médicament dans le pays si elle voulait toucher les droits. Dans le cadre du nouvel accord, elle pourra simplement importer le produit pour toucher les droits de brevets.

- Les brevets seront protégés pendant au minimum vingt ans à partir de la date du dépôt de la demande de brevet, et la protection des brevets existants sera prolongée à partir du moment où l'accord sera appliqué par le pays (article 33). Les licences obligatoires accordées par les pouvoirs publics avant le $1^{\text {er }}$ janvier 1995 ne seront pas soumises au régime de l'accord (article 70). Les droits conférés incluent le fait, pour les brevets de produits, d'empêcher «des tiers agissant sans le consentement du titulaire du brevet d'accomplir les actes ci-après: fabriquer, utiliser, offrir à la vente, vendre ou importer à ces fins ce produit» (article 28). Néanmoins, le droit exclusif conféré par cet article d'importer le produit breveté est atténué par l'article 6 de l'accord qui exclut du champ de son application la question de l'épuisement des droits de propriété intellectuelle. En bref, par ce biais un Etat est autorisé à importer, sans l'autorisation du titulaire de brevet, un produit breveté (importation parallèle) de n'importe quel Etat membre de l'OMC où il aurait été légalement commercialisé. Cette clause a pour objectif d'augmenter la concurrence puisque les mêmes produits n'ont pas le même prix dans tous les pays.

- L'accord TRIPS permet aux pays, lorsqu'ils élaboreront ou modifieront leurs lois, «d'adopter les mesures nécessaires pour protéger la santé publique et la nutrition (...) à condition que ces mesures soient compatibles avec les dispositions du présent accord» (article 8). En conséquence un Etat peut prévoir des exceptions lorsqu'il considère qu'elles sont nécessaires pour protéger la santé publique. De telles exceptions devront être limitées et justifiées.

- L'accord permet d'utiliser le brevet sans l'autorisation du titulaire du brevet (licence obligatoire) et énonce les conditions dans lesquelles cela est possible: les Etats peuvent déroger à ces conditions en cas d'urgence nationale, d'utilisation publique à des fins non commerciales et pour remédier à des pratiques anticoncurrentielles.

10. OMS/DAP, Mondialisation et accès aux médicaments - Les implications de l'accord ADPIC/OMC, Genève, OMS, publication en préparation, 1998. 
口 Enfin, l'accord TRIPS, qui est entré en vigueur le $1^{\text {er }}$ janvier 1996, devra être appliqué par les pays industrialisés au plus tard un an après cette date par les pays en développement, par les pays en transition cinq ans après $\left(1^{\mathrm{er}}\right.$ janvier 2000), et par les pays les moins développés onze ans après 2006 avec la possibilité de prorogation. Les pays en développement qui n'accordaient pas de brevets de produits avant la signature de l'accord auront dix ans pour assurer une telle protection. Pendant la période de transition, les pays devront mettre en place une structure pour recevoir les demandes de brevets pour les nouveaux médicaments (article 70.8); en attendant la délivrance du brevet, des droits exclusifs de commercialisation seront accordés pour cinq ans à partir de l'obtention de l'autorisation de mise sur le marché dans le pays (article 70.9).

\section{QUELLES CONSÉQUUNCES CE NOUVEL ACCORD AURA-T-IL SUR LA SITUATION PHARMACEUTIQUE MONDIALE ET SUR L'ACCÈS AUX MÉDICAMENTS DANS LES PVD?}

Les accords TRIPS ayant été créés de toutes pièces durant l'Uruguay Round, leurs effets n'ont pas encore pu être étudiés. Néanmoins on peut, à partir des expériences passées, tirer quelques conclusions sur les conséquences probables qu'ils auront dans le domaine du médicament et des politiques pharmaceutiques. Ces conséquences ne seront pas identiques pour les trois groupes de pays définis plus haut: les pays industrialisés, les pays en développement ayant une industrie pharmaceutique nationale et les autres pays en développement.

\subsection{PAYS INDUSTRIALISÉS}

Les profits accrus provenant d'une meilleure protection intellectuelle et de la disparition de produits qui étaient en compétition avec le médicament breveté devraient permettre aux firmes de recherche de financer plus de recherche, de découvrir de nouveaux médicaments et ainsi de renforcer leur position sur le marché international. Il est probable que la recherche continuera à s'orienter vers les problèmes de santé des pays développés. Certains de ces nouveaux médicaments, tels que les médicaments antirétroviraux pour traiter le sida, seront certainement utiles aussi bien au Sud qu'au Nord. Le cas du praziquantel montre néanmoins que la découverte d'un nouveau médicament n'entraîne pas forcément sa disponibilité pour les malades; cela est spécialement vrai pour les pauvres des pays en développement. D'autre part, en l'absence d'une demande solvable dans les pays du Sud, la recherche entreprise par les firmes transnationales ne s'orientera pas sans incitation vers les problèmes majeurs de ces pays.

\subsection{PAYS EN DÉVELOPPEMENT AYANT UNE INDUSTRIE PHARMACEUTIOUE NATIONALE}

Trois grands thèmes occupent le débat sur les avantages et les inconvénients de la protection intellectuelle pour ce type de pays. 


\section{$\square$ L'accord TRIPS va-t-il favoriser la recherche?}

Le coût de la recherche/développement pour une nouvelle molécule a été estimé par l'industrie pharmaceutique aux environs de 300 millions de dollars" firmes pharmaceutiques des pays en développement ont un volume de ventes suffisant pour réaliser de tels investissements. D'autre part, bien qu'aujourd'hui un certain nombre de grands pays en développement possèdent les compétences scientifiques pour produire par un procédé nouveau des produits déjà sur le marché, de nombreux auteurs s'accordent à penser que le succès dans le cadre de l'innovation, comme celle qui a lieu pour les nouveaux médicaments, est plus lié au niveau du développement économique d'un pays qu'à son système de protection de brevets. Aucun pays de niveau socio-économique intermédiaire avec une protection des brevets de produits n'a inventé de nouveaux médicaments $^{12}$. L'invention pharmaceutique semble être plus le résultat du développement économique qu'un facteur de ce développement. D'ailleurs, la plupart des pays développés ont adopté un système de brevet à un stade assez tardif de leur développement et l'industrie pharmaceutique a bénéficié et continue de bénéficier des efforts faits dans la recherche fondamentale.

A court terme, les firmes locales qui souhaiteraient produire et commercialiser des médicaments couverts par un brevet devront payer des redevances au titulaire du brevet et n'auront plus la possibilité de développer des activités de recherche pour découvrir de nouveaux procédés de fabrication: le cas du praziquantel montre que grâce à cette absence de protection du produit un plus grand nombre de malades atteints de schistosomiase a pu être soigné, les prix ayant baissé de façon sensible sur le marché mondial ${ }^{13}$. Dans le long terme, il est probable que quelques entreprises des grands pays en développement seront capables de relever le défi et de se lancer avec le soutien de leur gouvernement dans des activités de recherche conduisant à la découverte de nouvelles molécules ${ }^{14}$.

\section{$\square$ L'accord TRIPS va-t-il favoriser le transfert de technologie?}

Jusqu'à maintenant la plupart des législations nationales obligeaient une entreprise qui détenait un brevet à produire le médicament dans le pays ou à accorder des licences si elle voulait toucher ses droits. Importer ne suffisait pas. Avec les accords TRIPS, il suffira d'importer pour toucher les droits de brevets. Pour certains auteurs, les firmes pharmaceutiques vont préférer concentrer leur production et exporter plutôt que de fabriquer sur place ou d'accorder des licences ${ }^{15}$. Pour d'autres, la production va continuer à avoir lieu dans les pays en développement. Il est difficile aujourd'hui de prévoir ce qui va se passer; très probablement l'importance des marchés et le climat pour les investissements seront des facteurs déterminants du transfert de technologie.

11. FIIM/IFPMA, GATT TRIPs and the pharmaceutical industry: a review, Geneva, 1995.

12. Challú P.M. «The consequences of pharmaceutical product patenting», World Competition, 1991, vol. 15 (2), pp. 65-126.

Nogues J., «Social costs and benefits of introducing patent protection for pharmaceutical drugs in developing countries », The Developing Economies, March 1993, vol. 31 (1), pp. $24-53$.

Nogues J., «Patents and pharmaceutical drugs: Understanding the pressures on developing countries», Journal of World Trade, 1990, vol. 24 (6), pp. 81-104.

13. Reich M.R. et al., International strategies for tropical disease treatments: experiences with praziquantel. WHO/DAP/CTD/98.5, Genève, OMS, 1998.

14. Rapp R., Rozek R., «Benefits and costs of intellectual property protection in developing countries», Journal of World Trade, 1990, vol. 24, pp. 75-102.

15. Verma S.K., "TRIPs, development and transfer of technology», International Review of Industrial Property and Copyright Law, 1996, vol. 27 (3). 


\section{$\square$ L'accord TRIPS va-t-il augmenter les prix des médicaments?}

Un certain nombre de firmes locales préféreront concentrer leurs efforts sur la production de médicaments génériques (c'est-à-dire hors brevet); cette tendance pourrait conduire à une diminution des prix de ces produits. Par contre, le prix des produits protégés par des brevets va augmenter, car ils seront en position de monopole ${ }^{16}$. Comme dans la majorité des cas les brevets sont aux mains des firmes multinationales, les PVD se trouveront confrontés à des situations de monopole sur leur marché intérieur avec un transfert de rentes vers les pays industrialisés. Le peu d'informations disponibles suggèrent que les pays protégeant plus strictement les brevets transfèrent des rentes plus élevées que les pays qui ont un faible niveau de protection des brevets ${ }^{17}$. D'autre part, les diminutions importantes de prix qui ont lieu lors de l'introduction de médicaments génériques à la suite de l'expiration d'un brevet seront retardées avec l'augmentation de la durée des brevets.

\subsection{AUTRES PAYS EN DÉVELOPPEMENT}

Un certain nombre de conséquences prévisibles sur les prix des médicaments s'appliqueront aussi à ce groupe de pays. Comme pour les pays en développement du groupe précédent, l'accord TRIPS n'aura pas un impact immédiat et direct sur les politiques de médicaments essentiels, la plupart de ces médicaments étant hors brevet. Dans les années à venir néanmoins, le problème des nouveaux médicaments va se poser. Etant donné l'évolution de la consommation et le besoin en médicaments toujours plus sophistiqués, le coût pour les budgets de santé et pour les populations qui financent l'essentiel de leur consommation directement pourrait augmenter.

\section{UN DÉFI MAJEUR POUR LE FUTUR}

L'accord TRIPS représente une concession importante des pays en développement, tout au moins de quelques-uns d'entre eux, en échange de bénéfices obtenus par l'Uruguay Round dans son ensemble. Cet accord, s'il n'est pas appliqué avec discernement, peut avoir des conséquences négatives sur l'accès aux médicaments dans les pays en développement et remettre en cause les quelques progrès qui ont pu être constatés çà et là dans ces pays. Il est donc urgent que les gouvernements, les organisations internationales, les firmes pharmaceutiques et tous les autres acteurs de la scène pharmaceutique définissent ensemble de nouvelles stratégies qui assurent un accès équitable aux médicaments et à la santé tout en respectant les conditions posées par l'accord; cela est un défi majeur pour les années à venir. Les principaux axes de réflexion pourraient s'organiser autour des thèmes suivants:

๑ Comment accélérer la mise en œuvre de politiques pharmaceutiques appropriées dans les pays en développement, l'objectif principal de ces politiques

16. WHO/SEARO, The Uruguay Round and health, SEA/ECON/14, New Delhi, WHO Regional Office for South East Asia, 1996.

17. Institut universitaire d'études du développement, Effets de l'Uruguay Round sur les pays en développement: synthèse des études par pays, vol. 2, Genève, IUED, 1995, p. 28. 
étant de permettre la distribution la plus grande de médicaments essentiels de bonne qualité au coût le plus bas possible et leur usage rationnel ? La plupart des médicaments essentiels étant hors brevet (génériques) et donc à des prix compétitifs sur le marché international, les nouvelles provisions contenues dans l'accord TRIPS ne devraient pas avoir un effet immédiat sur les prix et la disponibilité de ces médicaments.

- Comment améliorer l'accès aux nouveaux médicaments (antirétroviraux, antituberculeux, etc.) qui ne seront disponibles que sous brevet à des prix relativement élevés, et quelles stratégies développer en collaboration avec l'industrie pharmaceutique basée sur la recherche pour que les populations démunies ne soient pas privées des fruits de la recherche pharmaceutique et que celle-ci s'oriente aussi vers les problèmes spécifiques des pays en développement?

- Quels mécanismes mettre en place dans les pays qui ont déjà une industrie pharmaceutique nationale pour orienter l'effort des industries et des gouvernements vers la recherche et le développement et créer une véritable capacité d'innovation pharmaceutique?

- Comment utiliser au mieux les possibilités que donnent l'accord et la période de transition pour introduire dans les législations nationales des clauses qui permettront de sauvegarder les intérêts de la santé publique? Il est en effet reconnu dans l'accord que des mesures nécessaires pour protéger la santé publique peuvent être adoptées par les pays à condition qu'elles soient consistantes avec les articles de l'accord.

- Enfin, pour que l'accord aille dans le sens de l'intérêt national et soit un moyen d'améliorer la situation pharmaceutique, il est nécessaire que les pays mettent en place un appareil administratif efficace chargé des problèmes de protection intellectuelle. Un tel appareil entraînera un surcoût de dépenses publiques dans des Etats déjà sans ressources; comment l'organiser et quel pourrait être le rôle de la communauté internationale dans ce domaine?

En conclusion, bien qu'il soit aujourd'hui difficile de mesurer avec certitude l'impact de l'accord TRIPS sur la situation pharmaceutique mondiale et sur l'accès des populations des pays en développement aux médicaments, il est clair que cet accord représente un nouveau défi pour la communauté internationale qui doit redoubler d'efforts afin d'améliorer la situation des quelque 2 milliards d'êtres humains qui n'ont pas accès aux médicaments les plus essentiels. 